\title{
Strategy to combat obesity and to promote physical activity in Arab countries
}

This article was published in the following Dove Press journal:

Diabetes, Metabolic Syndrome and Obesity:Targets and Therapy

7 March 2011

Number of times this article has been viewed

\author{
Abdulrahman O Musaiger' \\ Hazzaa M Al Hazzaa ${ }^{2}$ \\ Aayed Al-Qahtani ${ }^{3}$ \\ Jalila Elati ${ }^{4}$ \\ Jasem Ramadan ${ }^{5}$ \\ Nebal A AboulElla ${ }^{6}$ \\ Najat Mokhtar ${ }^{7}$ \\ Hashem A Kilani ${ }^{8}$
}

'Arab Center for Nutrition, Bahrain; ${ }^{2,3}$ King Saud University, Saudi Arabia, ${ }^{4}$ National Institute of Nutrition,

Tunisia; ${ }^{5}$ University of Kuwait, Kuwait; ${ }^{6}$ National Nutrition Institute, Egypt;

${ }^{7}$ Ibn Tofail University, Morocco; ${ }^{8}$ Sultan

Qaboos University, Oman
Correspondence:Abdulrahman O Musaiger

Head,Arab Center for Nutrition,

Manama-Bahrain

Tel +973- I 739625955

Fax +973-17346339

Email amusaiger@gmail.com

\begin{abstract}
Obesity has become a major public health problem in the Arab countries, creating a health and economic burden on these countries' government services. There is an urgent need to develop a strategy for prevention and control of obesity. The third Arab Conference on Obesity and Physical Activity was held in Bahrain in January 2010, and proposed the Strategy to Combat Obesity and Promote Physical Activity in Arab Countries. This strategy provides useful guidelines for each Arab country to prepare its own strategy or plan of action to prevent and control obesity. The strategy focused on expected outcomes, objectives, indicators to measure the objectives, and action needs for 9 target areas: child-care centers for preschool children, schools, primary health care, secondary care, food companies, food preparation institutes, media, public benefit organizations, and the workplace. Follow-up and future developments of this strategy were also included.
\end{abstract}

Keywords: obesity, physical activity, strategy, Arab countries

\section{Introduction}

Obesity has become an epidemic worldwide. The World Health Organization (WHO) has warned of the escalating epidemic of obesity that could put the population in many countries at risk of developing noncommunicable diseases. ${ }^{1}$ In the Arab countries, the prevalence of overweight and obesity has changed drastically during the past 3 decades. $^{2}$

Yusuf et $\mathrm{al}^{3}$ reported that the Middle East region has the second highest mean body mass index after North America. The Middle East region also has the second highest mean waist:hip ratio, after South America. It is well-known that obesity is an important risk factor for chronic noncommunicable diseases, especially cardiovascular disease, diabetes, high blood pressure, and some types of cancer. These diseases have become the main causes of morbidity, mortality, and disability in these countries, and cause more than $50 \%$ of total deaths. ${ }^{4}$

The growing prevalence of type 2 diabetes, cardiovascular disease, and some types of cancer is linked to obesity. The burden of these diseases is particularly high in Arab countries. The high risk of both diabetes and cardiovascular disease associated with obesity in Arabs may be due to a predisposition to abdominal obesity which can lead to the metabolic syndrome..$^{5-7}$

Using the International Diabetes Federation Criteria, the prevalence of the metabolic syndrome was found to be $45.5 \%$ in women and $30 \%$ in men living in Tunisia, higher than the rate of $28.7 \%$ found by WHO using the same criteria. ${ }^{8}$ In the past 20 years, the rate of obesity has tripled in developing countries that have been adopting 
a Western lifestyle involving decreased physical activity and overconsumption of energy-dense food. Such lifestyle changes are also affecting children in these countries. The highest prevalence of childhood overweight was found in Eastern Europe and the Arab Middle East. The few studies conducted in the Arab countries showed a very high prevalence of the metabolic syndrome among youth.

Rapid changes in dietary habits, lifestyle, and lack of physical activity have led to an increase in the prevalence of overweight and obesity in Arab countries and consequently an increase in chronic noncommunicable diseases. According to the available data in the Arab world, overweight and obesity are prevalent even in poorer countries, as well as in low-income groups within these countries, exacerbating the burden on health authorities in the prevention and control of these diseases. ${ }^{2,9}$

Statistics published in 2007 indicate that the prevalence of overweight and obesity has reached an alarming level, necessitating urgent action. The estimated prevalence of overweight and obesity in preschool children (less than 5 years) was $4 \%$ to $8 \%$. The prevalence is now $12 \%$ to $25 \%$ among school-age children (6 to 10 ), and $20 \%$ to $45 \%$ in adolescents (11 to 18). Among adults the prevalence of overweight and obesity is remarkable, ranging between $40 \%$ and $60 \%$ for men, and between $50 \%$ and $70 \%$ for women. ${ }^{2}$

Food habits have become poorer during the last 3 decades, with a decline in the intake of fruit, vegetables, whole grains, and legumes, but a sharp increase in the intake of foods rich in fat, sugar and salt. ${ }^{10,11}$ Data from WHO (Regional Office in Cairo) indicate that between $79 \%$ to $96 \%$ of adults in 6 Arab countries (Egypt, Jordan, Iraq, Kuwait, Saudi Arabia, and Syria) eat less than 5 servings of fruit and vegetables per day. ${ }^{4}$ The recommendation is 5 to 9 serving per day.

Several other negative changes in food habits in the region have contributed significantly to the increase in prevalence of obesity and chronic noncommunicable diseases. These include an increase in the intake of unhealthy fast food and sugary drinks, skipping breakfast, consumption of foods rich in calories and fat between meals, and a high percentage of people who consume foods outside the home, which exposes them to more high-calorie foods. ${ }^{12}$

A change in patterns of physical activity has enhanced the complexity of the problem. The current reliance on modern means of transport, and an increase in the number of hours spent watching television, using the internet, and playing computer games, and thus decreasing physical activity, is increasing a sedentary lifestyle in Arab societies. ${ }^{6}$ According to WHO statistics (Regional Office in Cairo) lack of physical activity in adults ranged from $33 \%$ to $86 \%$ in 7 Arab countries (Egypt, Iraq, Jordan, Kuwait, Saudi Arabia, Sudan and Syria). ${ }^{5}$

Scientific evidence shows that people can remain in good health even into old age if prevention and control of risk factors associated with chronic, noncommunicable diseases are established early enough, notably control of obesity and unhealthy eating habits and physical inactivity. Scientific research has also contributed to an understanding of the many factors concerning the role of nutrition, healthy behaviors, maintenance of a healthy weight, and physical activity in health promotion. Research has shown that physical activity has additional health benefits not related to nutrition, and is an essential means to improving physical, psychological, and social aspects of human health. ${ }^{1}$

Taking into account the above health and nutrition situation, there is a need to modify lifestyles including physical activity and dietary habits. Important aspects in this area can be summarized as follows:

1. Obesity has become a major health problem creating a health and economic burden on government services in Arab countries and, therefore, there is an urgent need to develop a strategy for prevention and control of obesity.

2. Lack of physical activity and inactivity is the most influential factor contributing to the high rates of obesity in Arab societies, and this requires development of effective programs to encourage physical activity.

3. Part of the solution to the problem involves changing dietary habits and another part involves changing lifestyle habits such as the amount of time spent watching television, using the internet and playing computer games.

4. There is a need to provide effective treatment services for obese people in health institutions.

\section{The strategy to combat obesity and promote physical activity in Arab countries}

This strategy was prepared by the Arab Taskforce for Obesity and Physical Activity, based in Bahrain, and presented at the Third Arab Conference on Obesity and Physical Activity, held in Bahrain during 19-21 January 2010. The conference was attended by participants from 14 Arab countries, and the strategy was discussed and approved by the participants in the closing session.

Because Arab countries have different socio-economic and political situations, this strategy could be modified to suit each country. In this way, the broad strategy can provide 
useful guidelines for national strategies to combat obesity in the Arab region. Timelines for the implementation of the strategy and the interim period measurements have not been provided, as these depend on the situation in each country, as well as the availability of base line data to implement the strategy.

\section{Vision}

Arab societies can enjoy good health through the promotion of healthy weight and physical activity which, in turn, will contribute to reducing morbidity, disability, and death resulting from chronic noncommunicable diseases.

\section{Duration}

This is a 5-year strategy (2010-2015).

\section{Goals}

1. To control and reduce the incidence of overweight and obesity in Arab societies by promoting health dietary habits and increased physical activity.

2. To reduce the risk factors for chronic noncommunicable diseases, which occur as a result of obesity, unhealthy diets, and lack of physical activity.

3. To raise awareness of Arab communities about the importance of physical activity, healthy nutrition, and the complications of obesity such as diabetes and cardiovascular disease, and maintaining a healthy weight, and the effect of these factors on improvement of health status.

4. To explore ways to develop partnerships between government institutions and the private sector and civil society for the purpose of promoting healthy lifestyles in Arab societies.

5. To establish ways of monitoring and assessing obesity and physical activity in Arab societies.

6. To conduct research on the health, nutrition, and social and economic factors associated with the occurrence of obesity and physical activity in Arab societies.

7. To provide effective treatment services to obese people.

\section{Elements of the strategy}

In order to achieve the strategy goals, activities should address the following aspects:

1. Focus on practical solutions that can be applied to combat obesity and promote physical activity.

2. At the initial phase the strategy should make use of the most reliable, available information rather than wait to collect surplus information.
3. Programs and activities should be long-term and sustainable, bearing in mind that changing unhealthy behaviors takes a great deal of time and effort. However, the strategy should incorporate programs that can be achieved in the short and medium term, and that contribute to reducing risk factors associated with obesity and physical activity.

4. The work plan and proposed activities should include all segments of society at all ages, taking into consideration their social and economic background.

5. Involvement of civil society and the private sector in addition to government institutions in the preparation and implementation of activities and proposed programs.

6. Disseminate and promote the positive benefits of healthy weight, physical activity, and healthy nutrition.

\section{Important factors for the success of the strategy}

1. The strategy programs and activities should be based on sound scientific evidence.

2. The strategy should take into account good practice from existing programs in other countries and, where appropriate, try to incorporate them to the local needs.

3. The relevant and concerned official bodies should endorse their commitment to implementing the strategy.

4. Strategic partnerships should be established between government institutions and the private sector and nongovernmental organizations, to prepare and implement proposed programs.

5. The development of new concepts, and new knowledge and skills should be supported by conducting studies, research, and capacity training.

\section{Responsibilities in the implementation of the strategy}

This strategy should encourage formulation and promotion of policies, plans, and programs in order to improve healthy nutrition, and encourage physical activity as well as maintaining a healthy weight. Given the significant variations in the socio-economic status of Arab countries, there is a need for regional and global cooperation in order to gain benefit from scientific and practical experiences.

Ministries of Health should have primary responsibility for coordinating and implementing the programs and plans of the strategy and higher national committee should be established with executive authority. This committee should be composed of representatives from all relevant government institutions, and representatives from the private sector and professional unions related to nutrition and physical activity. 


\section{Target areas}

Childcare centers for pre-school age children (including nurseries, kindergartens, and service centers for this age group)

\section{Expected outcomes}

1. Improvement of the environment and learning experiences of child care centers for pre-school age children, which will promote healthy nutrition and active play.

2. Use of these child care centers to improve the awareness, skills, and capacity of staff and parents to implement activities related to healthy nutrition, healthy weight, and active play.

3. Greater participation of children, parents, and the rest of the community in finding solutions to combat obesity in this age group.

\section{Objectives}

1. Increase the proportion of children of pre-school age who have active play for 30 minutes or more per day by $25 \%$.

2. Increase the proportion of children of pre-school age who eat a healthy breakfast at home by $25 \%$.

3. Increase awareness on the importance of physical activity and healthy nutrition among staff in these centers by $25 \%$.

4. Increase the proportion of children who eat 4 or more servings of fruit and vegetables by $25 \%$.

5. Reduce the proportion of overweight and obesity among these children by $25 \%$.

Indicators to assess achievement of the objectives

1. The proportion of children who engage in active play for more than 30 minutes a day.

2. The proportion of children and parents who eat a healthy breakfast daily at home.

3. The proportion of staff with knowledge and health behaviors associated with obesity and lifestyle habits related to preschool children in child care centers of pre-school age. This can be done by measuring the knowledge and behavior before and after the implementation of the strategy.

4. The proportion of children of pre-school age who eat 4 or more servings of fruit and vegetables a day.

5. The proportion of overweight and obesity in children before school age (using body mass index, in accordance with standards adopted by international organizations).

\section{Actions needed}

1. Prepare healthy food guides for pre-school age children for subsequent distribution to child care centers.

2. Prepare guidelines for active play for children of pre-school age and their distribution to child care centers.

3. Provide training courses and workshops for staff in these centers on how to promote physical activity, healthy lifestyles, and a healthy weight for children of pre-school age.

4. Establish regulations and pass legislation on adequate and safe spaces for active play in child care centers for pre-school age children.

5. Pass legislation on nutritional standards of food provided to children in these centers, as well as the handling and storage of these foods.

6. Provide lectures and workshops for parents on how to encourage physical activity and healthy nutrition in these children.

7. Monitor children enrolled in these centers, by measuring their height and weight, and recording dietary habits and physical activity.

\section{Schools (including primary and secondary schools in government and private sectors)}

\section{Expected outcomes}

1. Improvement of the school environment and educational activities related to the promotion of physical activity and healthy nutrition among students.

2. Promotion of physical activity and healthy nutrition for students, parents and local communities through schools and school facilities.

3. Increased participation of students, teachers and parents in finding practical solutions to encourage physical activity and healthy eating behaviors.

\section{Objectives}

1. Increase the proportion of children who eat a healthy breakfast daily at home by $25 \%$.

2. Increase the proportion of children who are physically active for one hour or more on 5 or more days of the week by $25 \%$.

3. Increase knowledge and skills of teachers in aspects related to the promotion of physical activity and measurement of obesity and nutritional status for school students by $25 \%$.

4. Reduce the percentage of students who watch television daily for more than 2 hours by $20 \%$.

5. Increase the percentage of students who eat at least 4 servings of fruits and vegetables per day by $25 \%$. 
6. Reduce the percentage of students who consume sugary drinks (eg, soft drinks, canned juices) more than 3 times per week by $20 \%$.

7. Reduce the percentage of students who eat unhealthy fast food more than 3 times per week by $30 \%$.

8. Increase the awareness of parents on the importance of physical activity and healthy nutrition for their school children by $50 \%$.

\section{Indicators to assess achievement of the objectives}

1. The proportion of students who eat a healthy breakfast daily at home.

2. The proportion of school students who are physically active for an hour or more per day.

3. The proportion of teachers who have appropriate knowledge and skills regarding physical activity, healthy nutrition, and for measuring obesity (such as using body mass index). This indicator can be established by measuring the knowledge and behavior before and after the implementation of the strategy.

4. The percentage of students who watch television for more than 2 hours or more a day.

5. The percentage of students who eat 4 or more servings of fruit and vegetables per day.

6. The percentage of students who consume sugary drinks more than 3 times a week.

7. The percentage of students who have correct knowledge about the importance of physical activity, healthy nutrition, and healthy weight.

8. The proportion of parents who have adequate knowledge, attitudes, and practices regarding the physical activity, healthy nutrition, and healthy weight of their children.

9. The percentage of students who eat unhealthy fast food more than 3 times a week.

\section{Actions needed}

1. Integrate activities and programs to promote physical activity and nutrition in the school health plan.

2. Integrate the concepts, information, and skills related to physical activity, healthy nutrition, and healthy weight in school curricula.

3. Develop legislation on health and nutritional aspects of food provided to the students in schools.

4. Provide training courses for teachers on how to promote physical activity and healthy nutrition in students.

5. Develop guidelines on the promotion of healthy nutrition for school students.
6. Prepare guidelines that support physical activity prescription of school students.

7. Conduct research on dietary, lifestyle, social, and psychological factors associated with obesity and physical activity in school students.

8. Take advantage of non-class activities to promote physical activity and healthy nutrition in school students.

9. Develop practical guidelines for teachers on prevention and control of obesity among school children.

10. Organize awareness programs for students on healthy nutrition and the importance of physical activity, and on the negative aspects of long periods spent watching television, playing computer games, and using the Internet.

11. Organize meetings and educational programs for parents on appropriate ways to promote physical activity and healthy nutrition and healthy weight in children.

\section{Primary health care (including health centers and maternal and child health centers)}

\section{Expected outcomes}

1. Enhancement of knowledge and skills related to promoting physical activity, healthy nutrition, and healthy weight among health staff in these centers.

2. Enhancement of the skills of primary health care physicians needed to measure, prevent and control obesity in different age groups.

3. Improvement of the knowledge of visitors to primary health centers on the advantages of healthy weight and physical activity.

4. Provision of treatment services for obese people in primary health centers.

\section{Objectives}

1. Increase by $50 \%$ the proportion of health staff in primary health care centers who have adequate knowledge and skills regarding physical activity, healthy nutrition, and healthy weight.

2. Increase by $50 \%$ the proportion of physicians working in primary health care centers who have adequate knowledge and skills in the assessment, control, and treatment of obesity.

3. Increase awareness of visitors to primary health care centers about the importance of physical activity, healthy nutrition, and healthy weight by $50 \%$. This increase can be assessed by measuring the knowledge and behavior before and after the implementation of the strategy. 
4. Increase by $40 \%$ in the number of mothers who breastfeed their infants during the first 6 months after childbirth.

\section{Indicators to assess achievement of objectives}

1. The proportion of health workers in primary health care centers who have adequate knowledge on promotion of physical activity, healthy nutrition and healthy weight.

2. The proportion of physicians working in primary health care centers who have adequate knowledge and skills in the assessment, control, and treatment of obesity.

3. The proportion of visitors to primary health care centers who have adequate knowledge about the importance to their health status of physical activity, healthy nutrition, and healthy weight.

4. The proportion of mothers who breastfeed their infants during the first 6 months of life.

\section{Actions needed}

1. Formulate and promote guidelines on healthy nutrition, lifestyle, physical activity, and healthy weight at different ages for visitors and staff in primary health care centers.

2. Organize training courses on measurement and control of obesity for physicians, nurses, and midwives working in primary health care centers.

3. Conduct activities and programs to increase the awareness of visitors to primary health care centers regarding healthy nutrition and healthy weight.

4. Create a system to monitor the weight and height of visitors and beneficiaries of primary health care centers.

5. Promote breastfeeding by implementing the code of the Marketing of Breastmilk Substitutes and the Baby Friendly Hospital and other programs proposed by international organizations.

6. Establish special clinics to treat obese people.

\section{Secondary care (including hospitals, treatment centers, and centers for obesity and other chronic diseases such as diabetes and heart disease)}

\section{Expected outcomes}

1. Improve the knowledge and skills related to the measurement and methods of treatment of obesity.

2. Establish programs for early detection of risk factors associated with obesity (diabetes, high blood pressure, blood lipids, and cardiovascular diseases).

\section{Objectives}

1. Increase the proportion of physicians who have adequate knowledge and skills to assess, control, and treat obesity in different age groups by $50 \%$.
2. Establish or increase the proportion of programs related to early detection of risk factors associated with obesity in hospitals by $50 \%$.

3. Increase awareness of patients attending hospitals about how to prevent and control obesity and associated risk factors by $25 \%$.

4. Provide treatment services for obese people.

Indicators to assess achievement of the objectives

1. The proportion of physicians working in government and private hospitals who have adequate skills to assess and control obesity.

2. Availability of early detection programs for risk factors in obese people.

3. The level of knowledge about prevention and treatment of obesity in patients attending hospitals. This can be done by measuring the knowledge and behavior before and after the implementation of the strategy.

4. The number of clinics in the hospitals that provide treatment services for obese people.

\section{Actions needed}

1. Conduct training programs and courses for physicians and stakeholders in hospitals on assessment, control, and treatment of obesity.

2. Establish a program for the early detection of obesity and associated risk factors.

3. Implement educational programs for patients and visitors to hospitals on the importance of controlling and treating obesity.

4. Establish multi-disciplinary clinics to control obesity.

\section{Food companies (including all the} institutions that manufacture and produce foods)

\section{Expected outcomes}

1. An increase in healthy choices for the community with food and beverages that contain fewer calories.

2. Availability of healthy foods that contain fewer calories in remote and poor areas, as well as cities.

3. More food companies that manufacture healthy, lowcalorie foods as well as small portions.

4. An increase in the proportion of families who consume low calorie foods outside the home.

5. More food companies that include nutritional information for their food products, specifically calorie and fat content.

6. Participation of food companies in programs to combat obesity and promote physical activity. 


\section{Objectives}

1. Increase the proportion of low-calorie foods and beverages available in food markets by $25 \%$.

2. Increase the proportion of low-calorie foods and beverages in remote and poor areas by $25 \%$.

3. Increase the number of food companies that manufacture low-calorie foods and in smaller portions by $25 \%$.

4. Increase the proportion of families who consume low calorie foods and smaller portions outside the home by $25 \%$.

5. Increase the proportion of food stuffs with nutritional information on their labels, especially the calorie and fat content by $25 \%$.

Indicators to assess the achievement of objectives

1. The proportion of low-calorie foods and beverages available in the food market.

2. The proportion of foods with smaller portion sizes available in the food market.

3. The proportion of the number of food companies that produce healthy foods with low calories and in smaller portions.

4. The proportion of families who consume low-calorie food outside the home.

5. The proportion of food stuffs with information on calorie and fat content on their labels.

\section{Actions needed}

1. Develop legislation to categorize the appropriate portion and calorie content of specific processed foods, especially relating to flavored milk, chocolate, sweets, and sugary drinks.

2. Develop legislation that enforces the addition of nutritional information on food labels to include adequate information on calories, composition, and portion size.

3. Develop a system to adopt, encourage, and reward food companies that offer healthy low-calorie foods.

4. Study the possibility of imposing more taxes on processed foods that contain higher-calories, especially sugary drinks.

\section{Food preparation and service institutions (including large restaurants, cafeterias and fast-food restaurants, especially those providing Western and local fast foods)}

\section{Expected outcomes}

1. Improve the availability of healthy low-calorie meals to the general public.

2. Increase the provision of healthy food choices in these institutions.
3. Improve the health and nutrition awareness of the owners and managers of these food institutions, as well as employees regarding the importance of healthy eating and maintenance of a healthy weight.

\section{Objectives}

1. Increase the number of healthy, low-calorie meals served in large restaurants, especially fast-food restaurants by $25 \%$.

2. Increase the provision of healthy food choices to the public in these institutions by $25 \%$.

3. Increase knowledge about the preparation of healthy, low-calorie foods among staff in these restaurants and cafeterias by $50 \%$.

Indicators to assess achievement of the objectives

1. The proportion of restaurants offering healthy meals low in calories and available in smaller portions.

2. Health awareness among restaurant workers about preparing healthy and low-calorie foods.

\section{Actions needed}

1. Establish guidelines on how to prepare healthy, lowcalorie foods for large food institutions that prepare and serve food to the public.

2. Conduct educational programs for workers in these food institutions on the importance of maintaining a healthy weight, and of healthy food preparation.

3. Develop legislation that encourages provision of adequate information on the calorie content of main meals on the menu.

4. Develop legislation that provides regulations to fast-food restaurants on recommended portion sizes and on healthy food preparation methods.

\section{Media and commercial promotion (including all means of communication and advertising as well as other means of promotion and marketing of food)}

\section{Expected outcomes}

1. The protection of society, particularly children, from misleading food advertisements and promotions, especially those that can relate to obesity.

2. Improve the level of knowledge and skills related to promoting physical activity, healthy nutrition, and healthy weight throughout society.

3. Protect society from the promotion of equipment, herbal preparations, pharmaceuticals, and other methods that have not been proven effective in the treatment of obesity. 


\section{Objectives}

1. Increase by $25 \%$ the percentage of programs channeled through the media, which relate to the importance of preventing obesity, and the promotion of physical activity and healthy nutrition.

2. Increase by $30 \%$ the proportion of food advertisements that contain accurate information and positive messages in the media.

3. Reduce by $30 \%$ the availability and promotion of equipment, herbal preparations, pharmaceuticals, and other methods accessible in the market, which have not been proven to be effective in treating obesity.

\section{Indicators to assess achievement of the objectives}

1. Percentage of programs delivered through the various media on promoting physical activity, healthy nutrition, and maintaining a healthy weight.

2 . The proportion of advertisements that contain negative messages related to healthy nutrition and healthy weight.

3. The proportion of members of the community who have adequate information about the prevention and control of obesity, and the need for physical activity to promote health status.

4. The number of institutions that sell equipment, herbal preparations, pharmaceuticals, and other methods that have not been proven effective in the treatment of obesity.

\section{Actions needed}

1. Develop specialized programs to promote physical activity and healthy nutrition for different age groups, as well as the assessment and control of obesity, especially television programs that target families.

2. Develop legislation targeting the texts and the contents of food advertisements broadcast through various media, to reduce the negative impacts of these advertisements, especially on children.

3. Conduct studies and research on the impact of the media on dietary behaviors and lifestyle in different segments of society.

\section{Public benefit organizations (including associations, institutions, and non- governmental organizations)}

\section{Expected outcomes}

An increase in active participation of these organizations in the preparation and implementation of programs to combat obesity and encourage physical activity and healthy nutrition.

\section{Objectives}

Increase the number of private and nongovernmental organizations that actively participate in government programs to combat obesity and promote physical activity by $50 \%$.

Indicators to assess achievement of the objectives The proportion of private and non-governmental organizations that contribute to programs promoting physical activity, healthy nutrition, and maintenance of health weight.

\section{Actions needed}

1. Invite private and non-governmental organizations to participate in the National Committee or sub-committees overseeing the implementation of this strategy.

2. Provide staff and volunteers in these organizations with training courses, workshops, and seminars on promoting physical activity, healthy nutrition, and control of obesity.

3. Use of expertise within these institutions to plan and implement this strategy.

\section{The workplace (including government, private institutions, and the private sector)}

\section{Expected outcomes}

Establishment of specific places for active utilization of break times and leisure time, and for providing access to healthy foods, in government bodies, private institutions, and in private sector organizations with more than 100 employees.

\section{Objectives}

1. To increase by $50 \%$ the proportion of enterprises with more than 100 employees which provide special places for active utilization of break time and leisure time, and gaining access to healthy foods for employees, to foster a healthy environment that encourages physical activity and appropriate nutrition.

2. Increase by $50 \%$ the knowledge and skills of employees in these institutions, on the importance of controlling obesity, and promoting healthy nutrition and physical activity.

Indicators to assess achievement of the objectives

1. The proportion of institutions where there is adequate provision of areas for physical activity and that provide access to healthy foods. 
2. The proportion of employees who have adequate knowledge related to the prevention of obesity, and healthy nutrition and physical activity. This can be done by measuring the knowledge and behavior before and after the implementation of the strategy.

\section{Actions needed}

1. Develop legislation that stipulates the availability of adequate space for active recreation in enterprises employing more than 100 people.

2. Develop guidelines for the quality and quantity of food provided to employees, as well as the quality of physical activity in these institutions, taking into account the nature of the work of individuals.

3. Conduct awareness programs for employees and staff in these institutions relating to the prevention and control of obesity, and the encouragement of physical activity and healthy nutrition.

\section{Follow-up and future developments}

1. This strategy will be distributed to health authorities, civil institutions, food companies, and the private sector in all Arab countries, as well as relevant international organizations.

2. The Arab Taskforce for Obesity and Physical Activity will conduct a series of workshops in Arab countries to explain the strategy and particular areas of application, depending on the specific social, economic, and political situation of each country.

3. The Arab Taskforce for Obesity and Physical Activity will prepare various guidelines on healthy nutrition and physical activity for pre-school children, school students, and adults to be discussed in workshops for the purpose of adoption by the official bodies in Arab countries.
4. The Arab Taskforce for Obesity and Physical Activity will assist in conducting studies and research related to this strategy in Arab countries that need such assistance.

5. The Arab Taskforce for Obesity and Physical Activity will hold periodic meetings and seminars for the purpose of follow-up activities and programs associated with this strategy.

\section{Disclosure}

The authors report no conflicts of interest in this work.

\section{References}

1. World Health Organization. Global Strategy on Diet, Physical Activity and Health. Geneva, Switzerland; 2004.

2. Musaiger AO. Overweight and Obesity in the Arab countries: The Need for Action. Technical Report, Bahrain Center for Studies Research, Bahrain; 2007.

3. Yusuf S, Hawken S, Ounpun S, et al. Obesity and the risk of myocardial infarction in 2700 participants from 52 countries: a case-control study. Lancet. 2005;366:1540-1649.

4. WHO/EMRO. Regional data on non-communicable diseases risk factors. World Health organization, Regional Office of East Mediterranean. Non-communicable diseases. Website: http//www.emr.who.int.ncd. Accessed April 10, 2010.

5. Al-Shayji IA, Akanji AO. Obesity indices and major component of metabolic syndrome in young adult Arab subjects. Ann Nutr Metab. 2004;48:1-7.

6. Kelishadi R. Childhood overweight, obesity and the metabolic syndrome in developing countries. Epidemiol Rev. 2007;29:62-76.

7. Hossain P, Kawar B, Elnahas M. Obesity and diabetes in the developing world a growing challenge. N Engl J Med. 2007;356:213-215.

8. Harzallah F, Alberti H, Ben Khalifa F. The metabolic syndrome in an Arab population: a first look at the new international Diabetes Federation Criteria. Diabetic Med. 2006;23:441-444.

9. Ng SW, Zaghlool S, Ali HI, Harrison G, Popkin BM. The prevalence and trends of overweight, obesity and nutrition-related non-communicable diseases in the Arab Gulf states. Obesity Rev. 2011;12:1-13.

10. FAO/WHO. Technical Consultation on National Food - Based Dietary Guidelines. WHO. Regional Office. Cairo, Egypt; 2006.

11. Musaiger AO. Diet and prevention of coronary heart disease in Arab Middle East countries. Med Principles Pract. 2002; 11(Suppl 2):9-16.

12. Arab Center for Nutrition. Nutrition Situation in the Arab Countries. Manama, Bahrain; 2009.

Diabetes, Metabolic Syndrome and Obesity: Targets and Therapy

\section{Publish your work in this journal}

Diabetes, Metabolic Syndrome and Obesity: Targets and Therapy is an international, peer-reviewed open-access journal committed to the rapid publication of the latest laboratory and clinical findings in the fields of diabetes, metabolic syndrome and obesity research. Original research, review, case reports, hypothesis formation, expert
Dovepress

opinion and commentaries are all considered for publication. The manuscript management system is completely online and includes a very quick and fair peer-review system, which is all easy to use. Visit http://www.dovepress.com/testimonials.php to read real quotes from published authors. 